\title{
Delayed risk stratification in differentiated thyroid cancer
}

Delaying risk stratification until 8-12 months after treatment for differentiated thyroid cancer (DTC) enables a more accurate prediction of final outcome than risk stratification on the basis of data available at the time of diagnosis, confirms a study from Italy.

\section{The ability to predict the} final outcome was superior when delaying risk stratification... 77

In general, DTCs are indolent tumors, which have a good prognosis. Castagna et al. observed that, in patients without evidence of disease at the time of the first follow-up (8-12 months) after total thyroidectomy and radioiodine ablation therapy, the risk of recurrence at subsequent examinations was very low, regardless of the initial risk stratification.

To confirm their observations, the investigators enrolled 512 patients with DTC whose risk of tumor recurrence or death was initially defined according to guidelines of the American and European Thyroid Associations (ATA and ETA, respectively). At 8-12 months after treatment, patients were restratified according to their clinical status.

Approximately $50 \%$ of patients initially in the intermediate to high risk group were classified as being at low risk using delayed risk stratification. On the other hand, about $10 \%$ of patients considered to be at low risk according to their initial risk assessment were redefined as being at high risk by delayed risk stratification. The ability to predict the final outcome was superior when delaying risk stratification than when using the ATA and ETA classifications.

The conclusions of Castagna et al. are consistent with previous results in patients with DTC stratified according to the response to therapy after 2 years of follow-up. "Our protocol has the advantage of reaching the same conclusions earlier on in the follow-up period," says senior investigator Furio Pacini, a Professor at the University of Siena.
The findings by Castagna et al. have important clinical implications, in particular for patients considered to be at high risk according to staging performed at diagnosis, when only pathological and demographics data are available. "Using delayed risk stratification, a substantial number of patients at intermediate to high risk will be redefined as being at low risk, just 8-12 months after initial treatment," explains Pacini. "Hence, in subsequent years, these individuals will be submitted to a less aggressive diagnostic follow-up."

Taken together, the results by Castagna and colleagues support a risk-adapted approach for the treatment of DTC, in which the risk estimate is continually adapted to the results of treatment.

\section{Linda Koch}

Original article Castagna, M. G. et al. Delayed risk stratification, to include the response to initial treatment (surgery and radioiodine ablation), has better outcome predictivity in differentiated thyroid cancer patients. Eur. J. Endocrinol. doi:10.1530/EJE-11-0466 